\title{
Exact Solution for Non-Self-Similar Wave-Interaction Problem during Two-Phase Four-Component Flow in Porous Media
}

\author{
S. Borazjani, ${ }^{1}$ P. Bedrikovetsky, ${ }^{1}$ and R. Farajzadeh ${ }^{2,3}$ \\ ${ }^{1}$ Australian School of Petroleum, The University of Adelaide, SA 5005, Australia \\ ${ }^{2}$ Shell Global Solutions International, Rijswijk, The Netherlands \\ ${ }^{3}$ Delft University of Technology, The Netherlands
}

Correspondence should be addressed to S. Borazjani; sara.borazjani@adelaide.edu.au

Received 6 September 2013; Revised 27 December 2013; Accepted 29 December 2013; Published 12 March 2014

Academic Editor: Shuyu Sun

Copyright (C) 2014 S. Borazjani et al. This is an open access article distributed under the Creative Commons Attribution License, which permits unrestricted use, distribution, and reproduction in any medium, provided the original work is properly cited.

\begin{abstract}
Analytical solutions for one-dimensional two-phase multicomponent flows in porous media describe processes of enhanced oil recovery, environmental flows of waste disposal, and contaminant propagation in subterranean reservoirs and water management in aquifers. We derive the exact solution for $3 \times 3$ hyperbolic system of conservation laws that corresponds to two-phase fourcomponent flow in porous media where sorption of the third component depends on its own concentration in water and also on the fourth component concentration. Using the potential function as an independent variable instead of time allows splitting the initial system to $2 \times 2$ system for concentrations and one scalar hyperbolic equation for phase saturation, which allows for full integration of non-self-similar problem with wave interactions.
\end{abstract}

\section{Introduction}

Exact self-similar solutions of Riemann problems for hyperbolic systems of conservation laws and non-self-similar solutions of hyperbolic wave interactions have been derived for various flows in gas dynamics, shallow waters, and chromatography (see monographs [1-8]). For flow in porous media, hyperbolic systems of conservation laws describe twophase multicomponent displacement $[9,10]$. Consider

$$
\begin{gathered}
\frac{\partial s}{\partial t}+\frac{\partial f(s, c)}{\partial x}=0 \\
\frac{\partial(c s+a(c))}{\partial t}+\frac{\partial(c f(s, c))}{\partial x}=0
\end{gathered}
$$

where $s$ is the saturation (volumetric fraction) of aqueous phase and $f$ is the water flux. Equation (1) is the mass balance for water and (2) is the mass balance for each component in the aqueous solution. Under the conditions of thermodynamic equilibrium, the concentrations of the components adsorbed on the solid phase $\left(a_{i}\right)$ and dissolved in the aqueous phase $\left(c_{i}\right)$ are governed by adsorption isotherms:

$$
a=a(c), \quad a=\left(a_{1}, a_{2}, \ldots, a_{n}\right), \quad c=\left(c_{1}, c_{2}, \ldots, c_{n}\right) .
$$

Exact and semianalytical solutions of one-dimensional flow problems are widely used in stream-line simulation for flow prediction in three-dimensional natural reservoirs [10]. The sequence of concentration shocks in the one-dimensional analytical solution is important for interpretation of laboratory tests in two-phase multicomponent flow in natural reservoir cores.

The scalar hyperbolic equations (1) and (2), $n=0$, correspond to displacement of oil by water $[9,10]$. The $(n+1) \times(n+1)$ system (1) and (2) describes two-phase flow of oleic and aqueous phases with $n$ components (such as polymer and different salts) that may adsorb and be dissolved in both phases. These flows are typical for so-called chemical enhanced oil recovery displacements, like injections of polymers or surfactants, and for numerous environmental flows $[9,10]$. For polymer injection in oil reservoirs, $i=1$ corresponds to polymer and $i=2,3, \ldots, n$ to different ions. 
Therefore the system (1) and (2) is called the multicomponent polymer-flooding model $[11,12]$. Besides, $(n-1) \times(n-1)$ hyperbolic system (1) and (2) describes two-phase $n$-component displacement, which is typical for so-called gas methods of enhanced oil recovery $[9,10,13,14]$. The processes of hot water injection with phase transitions, secondary migration of hydrocarbons with consequent formation of petroleum accumulations, enhanced geothermal energy projects, and injections into aquifers are described by the above systems. The Riemann problems correspond to continuous injection of chemical solutions or gases into oil reservoirs; the solutions are self-similar $[3,9,14]$. The wave-interaction problems correspond to piece-wise-constant initial-boundary conditions, for which the solutions are non-self-similar $[1,10,15-17]$. The wave-interaction solutions describe injection of limited slugs (banks) of chemical solutions or gaseous solvents driven in the reservoirs by water or gas $[9,10]$.

Riemann problem (1) and (2) with $n=1$ has been solved with applications to various injections of polymers $[17,18]$, carbonized water and surfactants $[19,20]$, and so forth. More complex self-similar solutions of (1) and (2) for $n=2,3$ were obtained by Barenblatt et al. [21] and Braginskaya and Entov [22] and later by Johansen et al. and Winther et al. [11, 12, 2325]. Analogous solutions for gas injection and $n=3,4, \ldots$ have been obtained by Orr and others $[9,13,26-31]$.

The system (1) and (2) describes two-phase multicomponent displacements in large scale approximation, where the dissipative effects of capillary pressure, diffusion, and thermodynamic nonequilibrium are negligible if they are compared with advective fluxes under the large length scale of the natural subterranean reservoirs. Travelling waves near to shock discontinuities in dissipative systems have been presented in $[10,32]$. A semianalytical global solutions have been obtained by Geiger et al. [33] and Schmid et al. [34]; see also [16].

The particular case of so-called multicomponent polymer flooding is the dependency of the component sorption concentration of its own concentration only $a_{i}\left(c_{1}, c_{2}, \ldots, c_{n}\right)=$ $a_{i}\left(c_{i}\right)$. Exact solutions of the Riemann problem for this case show that the concentration of each component performs the jump without shocks of other components (see the corresponding solution in the books $[10,21])$. Therefore, in concentration profiles, the shocks are located in order of decrease of derivatives of the sorption functions. In the case of Henry isotherms $a_{i}\left(c_{i}\right)=\Gamma_{i} c_{i}$, the shocks are located in order of increase of Henry's sorption coefficients $\Gamma_{i}$.

The distinguished invariant feature of $(n+1) \times(n+$ 1) conservation law systems for two-phase multicomponent flows in porous media with sorption and phase transitions equations (1) and (2) is its splitting into an $n \times n$ auxiliary system for concentrations $c_{i}(x, t)$ and a scalar hyperbolic equation for saturation $s(x, t)[35,36]$. This splitting explains the simple form of Riemann problem solutions for system (1) and (2) as compared with gas dynamics or chromatography $[1,2,37]$.

The non-self-similar solution of system (1) and (2), $n=$ 2, for slug injections has been considered by Fayers [17], where the qualitative behaviour of characteristic lines and shocks has been described. The exact solutions of system
(1) and (2) for $n=2$ and 3 have been obtained in [15] (see book [10] for detailed derivations, in which the sorption of component depends on its own concentration only $a_{i}=$ $\left.a_{i}\left(c_{i}\right), i=1,2, \ldots\right)$. Numerous interactions of different saturation-concentration shocks occur after the injection, resulting in appearance of moving zones with different combinations of components. However, after all interactions, different component slugs are separated from each other. As in the case of continuous injection, the slugs are finally positioned in the order of decreasing sorption isotherm derivatives $\left(d a_{i} / d c_{i}\right)$. It seems that this simplified case draws the line where the analytical solutions can be found from the analysis of system (1) and (2) directly. Consideration of crosseffects $a_{i}=a_{i}\left(c_{1}, c_{2}, \ldots, c_{n}\right)$ in sorption functions equation (3) introduces significant difficulties into wave analysis, and even the Riemann problem cannot be solved for any arbitrary case $n=2$ (see [38], where the Riemann solutions have been obtained for several particular cases).

The splitting technique reduces number of equations in (1) and (2) by one, allowing for exact solutions in more complex multicomponent cases [35-40]. The Riemann problem with cross-effects for adsorption $a_{i}=a_{i}\left(c_{1}, c_{2}\right)$ has been solved in $[39,41,42]$ for continuous polymer injection with varying salinity using the splitting method. In the current paper, the exact solution for non-self-similar problem of injection of polymer slug with varying salinity followed by water drive is obtained.

The structure of the text is as follows. The particular case of the general system (1) and (2) that is discussed in the current work is introduced in Section 2 along with physics assumptions and initial-boundary conditions for slug injection problem. The detailed description of the splitting procedure for the system is discussed along with formulation of initial and boundary conditions for the auxiliary system which is presented in Section 3. Section 4 contains derivation of the Riemann solution that corresponds to the first stage of the slug injection. The wave-interaction slug injection problem is solved in Section 5. Section 6 contains a simplified solution for the particular case where the initial chemical concentration is zero, which corresponds to the case of polymer slug injection. The paper is concluded by physical interpretation of the solution obtained for chemical slug injection with different water salinity into oilfield (Sections 7 and 8$)$.

\section{Formulation of the Problem}

Let us discuss the displacement of oil by aqueous chemical solution with water drive accounting for different salinities of formation and injected waters. In the following text, the component $n=1$ is called the polymer, and that $n=2$ is called the salt. The assumptions of the mathematical model are as follows: both phases are incompressible, dispersion and capillary forces are neglected, there are two phases (oleic and aqueous phases) and two components dissolved in water (polymer and salt), water and oil phases are immiscible, chemical and salt concentrations in water are negligibly small and do not affect the volume of the aqueous phase, 
the fractional flow of the aqueous phase is affected by concentration of the dissolved chemical, the fractional flow is independent of salt concentration, chemical and salt do not dissolve in oil, linear sorption for the polymer $a=\Gamma c$, Henry's sorption coefficient $\Gamma$ is salinity-dependent, salt does not adsorb on the rock, and temperature is constant.

The system of governing equations consists of mass balance equations for aqueous phase, for dissolved and adsorbed chemical, and for dissolved salt $[8,9]$ :

$$
\begin{gathered}
\frac{\partial s}{\partial t}+\frac{\partial f(s, c)}{\partial x}=0 \\
\frac{\partial(c s+a(c, \beta))}{\partial t}+\frac{\partial(c f(s, c))}{\partial x}=0 \\
\frac{\partial(\beta s)}{\partial t}+\frac{\partial(\beta f(s, c))}{\partial x}=0,
\end{gathered}
$$

where $s$ is the water saturation, $f$ is the fractional flow function, $a$ is the polymer sorption isotherm, and $c$ and $\beta$ are chemical and salt concentrations, respectively.

The fractional flow function (water flux) depends on the water saturation $s$ and on the chemical concentration $c$. The typical S-shapes of fractional flow functions $f$ under $c=$ const are shown in Figure 1. The fractional flow is a monotonically decreasing function of $c$. Sorption isotherms are linear for fixed salinity $a(c, \beta)=\Gamma(\beta) c$. The functions $f$ and $a$ are assumed to be bounded and smooth.

The system (4)-(6) is a hyperbolic $3 \times 3$ system of conservation laws with unknowns $s, c$, and $\beta$.

The displacement of oil by chemical slug corresponds to the following initial-boundary problem:

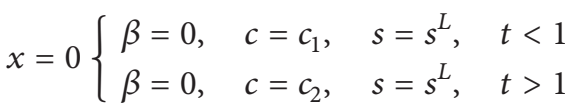

$$
\begin{aligned}
& t=0, \quad \beta=1, \quad c=c_{2}, \quad s=s^{R} .
\end{aligned}
$$

For $t<1$, during continuous injection of chemical solution with different salinity, the solution of system (4)-(6) subject to initial-boundary conditions equations (7) and (8) coincides with the solution of the Riemann problem:

$$
\begin{aligned}
& x=0, \quad \beta=0, \quad c=c_{1}, \quad s=s^{L} \\
& t=0, \quad \beta=1, \quad c=c_{2}, \quad s=s^{R} .
\end{aligned}
$$

The initial condition is denoted by $R$ in Figure 1 and the boundary condition corresponding to injection of the slug is denoted as $L$.

Generally $c(x, 0)=c_{2}>0$ is positive. Further in the text, the component with concentration $c$ is called "chemical," while for the case of the absence of this component initially in the reservoir $c(x, 0)=c_{2}=0$ we use the term "polymer."

The solution of the Riemann problem is self-similar: $s(x, t)=s(\xi), c(x, t)=c(\xi), \beta(x, t)=\beta(\xi), \xi=x / t$ and it can be found in $[37,39,40]$. The solution of the problem (7) and (8) in the neighbourhood of the point $(0,1)$ in $(x, t)$ plane is also self-similar. The global solution of the system (4)-(6) subject to the initial-boundary conditions equations

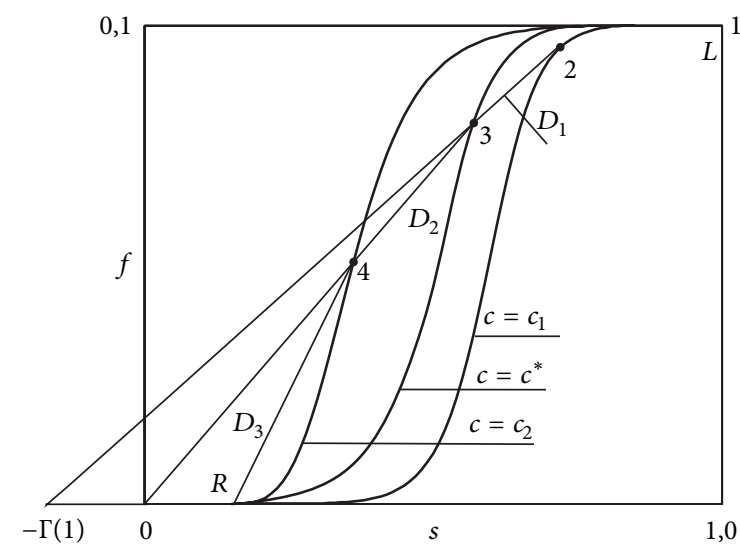

FIGURE 1: Fractional flow curves and Riemann problem solution, where $c_{1}$ is the slug concentration, $c_{2}$ is the initial concentration, and $c^{*}$ is the intermediate concentration.

(7) and (8) is non-self-similar; it expresses the interactions between hyperbolic waves occurring from decays of Riemann discontinuities in points $(0,0)$ and $(0,1)$ in $(x, t)$-plane.

System of (4)-(6) subject to the initial and boundary conditions equations (9) and (10) is solved in Section 4 using the method so-called splitting procedure [35]. This procedure is explained in the next section.

\section{Splitting Procedure}

In the present section we briefly explain the splitting method for the solution of hyperbolic system of conservation laws equations (4)-(6).

3.1. Streamline/Potential Function and Auxiliary System. As it follows from divergent (conservation law) form of equation for mass balance for water (1) or (4), there does exist such a potential function $\varphi(x, t)$ that

$$
\begin{aligned}
& s=-\frac{\partial \varphi}{\partial x} \\
& f=\frac{\partial \varphi}{\partial t} ;
\end{aligned}
$$

that is,

$$
\begin{gathered}
d \varphi=f d t-s d x, \\
\varphi(x, t)=\int_{0,0}^{x, t} f d t-s d x .
\end{gathered}
$$

Equation (4) is merely the condition of equality of second derivatives of the potential $\varphi$ as taken in different orders. It also expresses that the differential of the first order form equation (12) equals zero. The splitting procedure consists of changing the independent variables from $(x, t)$ to $(x, \varphi)$ in system (4)-(6). Figures 2 and 3 show the corresponding mapping $[43,44]$.

From fluid mechanics point of view, $\varphi(x, t)$ is a potential function, which equals the volume of fluid flowing through 

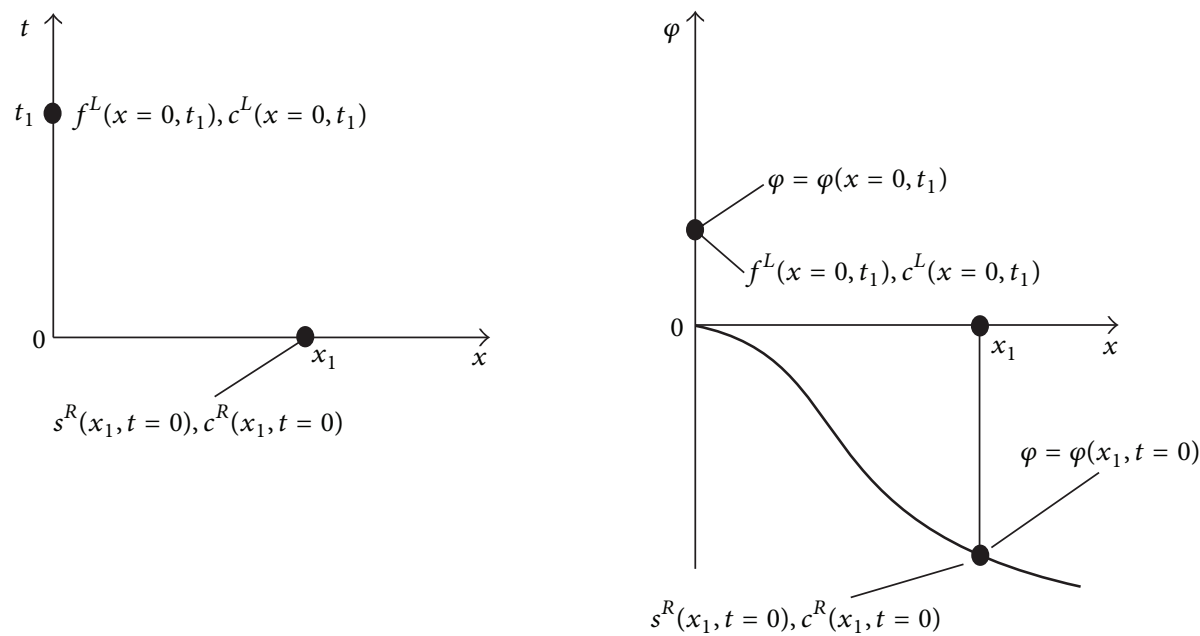

FIGURE 2: Introduction of potential function (Lagrangian coordinate) and mapping between independent variables.

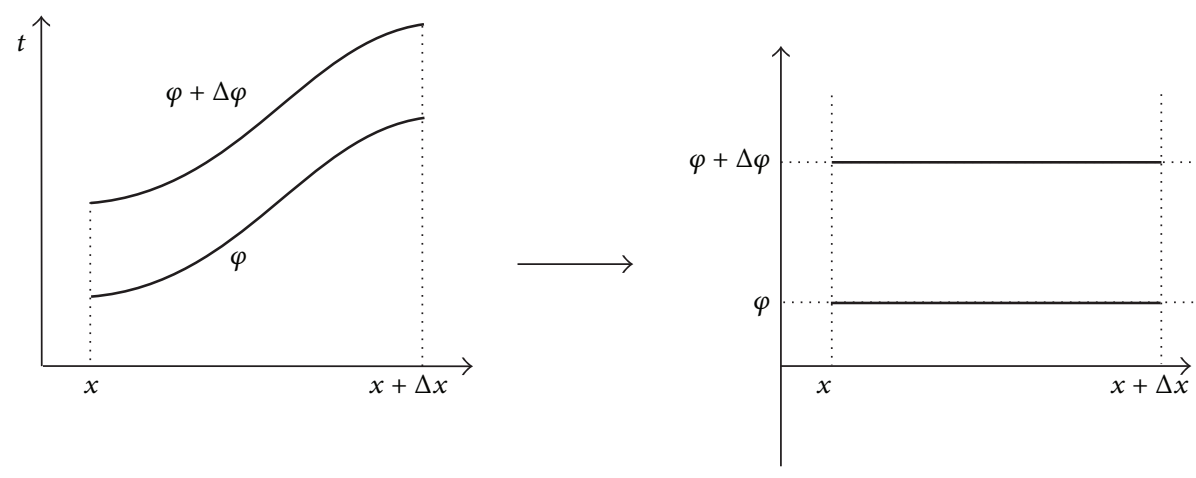

FIGURE 3: Derivation of mass balance equation in Eulerian and Lagrangian coordinate systems.

a trajectory connecting points $(0,0)$ and $(x, t)$. As it follows from (12), two streamlines in Figure 3 correspond to constant values of potential, that is, there is no flux through streamlines:

$$
\varphi\left(x, t_{2}\right)-\varphi\left(x, t_{1}\right)=\int_{t_{1}}^{t_{2}} f(x, t) d t
$$

Equation (4) shows that the integral of (13) along the closed contour is equal to zero; that is, the volume of fluid flowing through a trajectory connecting points $(0,0)$ and $(x, t)$ is independent of trajectory and depends on end points only. The potential function equation (13) is determined in the way that one end of trajectory is fixed at point $(0,0)$.

Let us derive the relationship between the elementary wave speeds of the system in $(\varphi, x)$ coordinates and those of the large system in $(t, x)$. Consider the trajectory $x=x_{0}(t)$ and its image $\varphi=\varphi_{0}(t)$ by the mapping equation (13):

$$
\varphi_{0}(t)=\varphi\left(x_{0}(t), t\right) \text {. }
$$

Define the trajectory speeds as

$$
D=\frac{d x}{d t}, \quad V=\frac{d x}{d \varphi} .
$$

Let us use $x$ as a parameter for both curves $x=x_{0}(t)$ and $\varphi=\varphi_{0}(t)$. Taking derivation of both parts of (13) in $x$ along trajectories and using speed definitions in (16), we obtain

$$
\frac{1}{V}=\frac{f}{D}-s
$$

from which follows the relationship between elementary wave speed in planes $(x, t)$ and $(x, \varphi)$ :

$$
D=\frac{f}{s+1 / V}
$$

For example, the eigenvalues of the system of equation in $(t, x)$ plane $\lambda_{i}$ and in $(\varphi, x), \Lambda_{i}$, are related by (Figure 4 $[43,44])$

$$
\Lambda_{i}(s, c)=\frac{f}{s+1 / \lambda_{i}} .
$$

From now on, the independent variables $(x, \varphi)$ are used in (4)-(6) instead of $(x, t)$. Expressing the differential form $d t$ from (12) as

$$
d t=\frac{d \varphi}{f}+\frac{s d x}{f}
$$



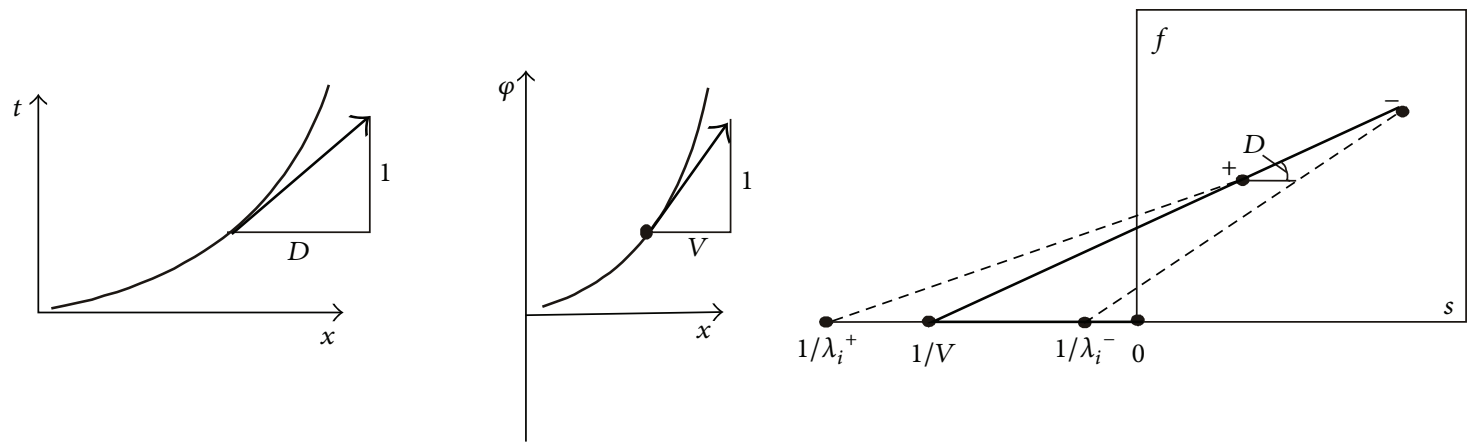

FIGURE 4: Speeds of a particle in Eulerian and Lagrangian coordinates.

and accounting for zero differential of the form $d t$

$$
d^{2} t=0=\left[\frac{\partial}{\partial x}\left(\frac{1}{f}\right)-\frac{\partial}{\partial \varphi}\left(\frac{s}{f}\right)\right] d x d \varphi
$$

we obtain the expression for (4) in coordinates $(x, \varphi)$

$$
\frac{\partial(s / f)}{\partial \varphi}-\frac{\partial(1 / f)}{\partial x}=0 .
$$

So, (22) is the mass balance for water; that is, it is (4) rewritten in coordinates $(x, \varphi)$.

Let us derive $(5)$ in $(x, \varphi)$ coordinates. The conservation laws for (5) in the integral form are

$$
\oint_{\partial \Omega}(c f) d t-(c s+a) d x=0
$$

where $\Omega$ is a closed domain $\Omega \subset R^{2}$, so the integral of (23) is taken over the closed contour.

Applying the definition of the potential function equation (13) into (23) yields

$$
\oint_{\partial \Omega} c(f d t-s d x)-a d x=\oint_{\partial \Omega} c d \varphi-a d x=0 .
$$

Tending the domain radius to zero and applying Green's theorem,

$$
\frac{\partial a(c, \beta)}{\partial \varphi}+\frac{\partial c}{\partial x}=0
$$

Now let us perform change of independent variables in (6) in $(x, \varphi)$ coordinates as follows:

$$
\begin{aligned}
\iint\left(\frac{\partial(\beta s)}{\partial t}+\frac{\partial(\beta f(s, c))}{\partial x}\right) d x d t & =\oint_{\partial \Omega} \beta s d x-\beta f(s, c) d t \\
& =\oint \beta d \varphi=\iint \frac{\partial \beta}{\partial x}=0 .
\end{aligned}
$$

Finally, the $(n+1) \times(n+1)$ system of conservation laws for two-phase $n$ component chemical flooding in porous media with adsorption can be split into an $n \times n$ auxiliary system equations (25) and (26) and one independent lifting equation
(22). The splitting is obtained from the change of independent variables $(x, t)$ to $(x, \varphi)$. This change of coordinates also transforms the water conservation law into the lifting equation. The solution of hyperbolic system (22), (25), and (26) consists of three steps: (1) solution of the auxiliary problem, (25), and (26) subject to initial and boundary conditions, (2) solution of the lifting equation, (22), and (3) determining time $t$ for each point of the plane $(x, \varphi)$ from (13).

The auxiliary system contains only equilibrium thermodynamic variables, while the initial system contains both hydrodynamic functions (phase's relative permeabilities and viscosities) and equilibrium thermodynamic variables.

The above splitting procedure is applied to the solution of displacement of oil by polymer slug with alternated salinity in the next section.

3.2. Formulation of the Splitting Problem for Two-Phase Flow with Polymers and Salt. Introducing new variables "density" $F$ and "flux" $U$ and applying the splitting technique, the $3 \times 3$ system (4)-(6) is transformed to the following form:

$$
\begin{gathered}
F=-\frac{s}{f}, \quad U=\frac{1}{f} \\
\frac{\partial(F(U, c))}{\partial \varphi}+\frac{\partial(U)}{\partial x}=0 \\
\frac{\partial a(c, \beta)}{\partial \varphi}+\frac{\partial c}{\partial x}=0 \\
\frac{\partial \beta}{\partial x}=0 .
\end{gathered}
$$

The auxiliary system equations (29) and (30) are independent of (28). The auxiliary system has thermodynamic nature since it contains only sorption function $a(c, \beta)$ and the unknowns are the component concentrations $c$ and $\beta$. Equation (28) is the volume conservation for two immiscible phases. For the known auxiliary solution of (29) and (30), equation (28) is a scalar hyperbolic equation. Figure 5 shows the projection of the space of the large system into that of auxiliary system and the lifting procedure $[43,44]$. 


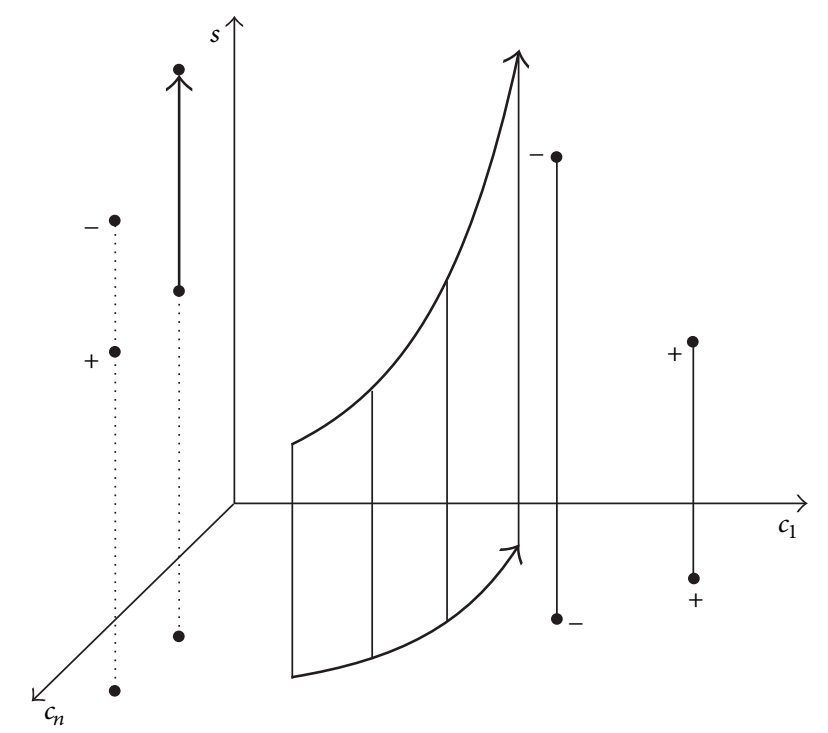

Figure 5: Projection of the space of the large system into that of auxiliary system and the lifting procedure using the solution of auxiliary system.

The boundary conditions for slug problem equation (7) are reformulated for coordinates $(x, \varphi)$ as

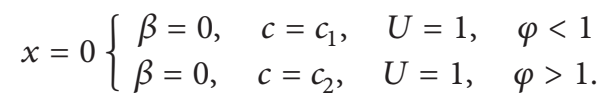

Figure 2 shows how the initial and boundary conditions for the large system (4) and (6) are mapped into those for auxiliary system and the lifting equations (28)-(30).

The initial conditions for slug problem equation (8) are reformulated for coordinates $(x, \varphi)$ as

$$
\varphi=-s^{R} x, \quad \beta=1, \quad c=c_{2}, \quad U=+\infty .
$$

The solution of the Riemann problem for $\varphi<1$ corresponds to the following initial and boundary conditions:

$$
\begin{gathered}
x=0, \quad \beta=0, \quad c=c_{1}, \quad U=1 \\
\varphi=-s^{R} x, \quad \beta=1, \quad c=c_{2}, \quad U=+\infty .
\end{gathered}
$$

\section{Solution for the Riemann Problem}

Let us discuss the solution of the problem equations (7) and (8) for $t<1$, which is self-similar; that is, the boundary and initial conditions become (9) and (10).

The mass balance conditions on shocks which follow from the conservation law (Hugoniot-Rankine condition) form of the system (28)-(30) are

$$
\begin{gathered}
\sigma[U]=[F] \\
\sigma[c]=[a] \\
\sigma[\beta]=0,
\end{gathered}
$$

where $\sigma$ is reciprocal to the shock velocity of (28)-(30). As salt and polymer concentration are connected by the thermodynamic equilibrium relationship $a(c, \beta)$, function $a$ is discontinuous if $c$ is discontinuous, so is $\beta$. Since $F$ is a function of $c$ and $U$, discontinuity of $c$ and $U$ yields discontinuity of $F$.

As it follows from equality (36), either $\sigma=0$ or $[\beta]=0$. From (34) and (35) it follows that if $\sigma=0,[a]=0$ and $[F]=0$. If $[\beta]=0$, from (35) and (36) it follows that $\sigma=[a] /[c]$ and $\sigma=[F] /[U]$; therefore it yields to $\sigma=[a] /[c]=[F] /[U]$. Finally from (34), if $[\beta]=0$ and $[c]=0$ this leads to $\sigma=$ $[F] /[U]$.

The shock waves must obey the Lax evolutionary conditions $[1-4,9]$.

4.1. Solution for the Auxiliary System. The solution of auxiliary system is presented in Figure 6 by sequence of $c$-shock from point $L$ into intermediate point and $(c, \beta)$-shock into point $R$. The corresponding formulae are as follows:

$$
\begin{aligned}
& c(x, \varphi) \\
& \beta(x, \varphi)
\end{aligned}= \begin{cases}c_{1}, & \beta=0, \quad \varphi>\Gamma(0) x \\
c^{*}, & \beta=0, \quad 0<\varphi<\Gamma(0) x \\
c_{2}, & \beta=1, \quad-s^{R} x<\varphi<0,\end{cases}
$$

where the condition of continuity of function $a(c, \beta)$ on the shock with $\sigma=0$, and (35) allows finding the intermediate concentration

$$
c^{*}=\frac{\Gamma(1)}{\Gamma(0)} c_{2}
$$

4.2. Solution for the Lifting Equation. Figure 7 exhibits initial and boundary conditions for hydrodynamics lifting equation (28). Curves $F=F(U, c)$ are shown for constants $c=c_{1}, c=$ $c_{2}$, and $c=c^{*}$; they are obtained from fractional flow curves $f=f(s, c)$ for the same constant values of concentration $c$. Point $R$ corresponds to $U$ tending to infinity and $F$ tending to minus infinity, where the fractional flow $f$ tends to zero. The tangent of the segment $(0,0)-(U, F)$ tends to $-s^{R}$.

The solution of lifting equation with known concentrations (37) is given by centred wave $L-2,(c-U)$-shock 2$>3$, $(\beta-c-U)$-shock $3->4$, and $U$-shock $4->R$ (Figure 7$)$. The centred wave $(L-2)$ is given by (39)

$$
\frac{\varphi}{x}=\frac{\partial F\left(U^{1}, c_{1}\right)}{\partial U} .
$$

Points 2 and 3 are determined by the condition of equality of $U$ and $c$ shock speeds:

$$
\frac{\partial F\left(U_{2}, c_{1}\right)}{\partial U}=\frac{F_{2}\left(U_{2}, c_{1}\right)-F_{3}\left(U_{3}, c^{*}\right)}{U_{2}-U_{3}}=\Gamma(0) .
$$

Point 4 is determined by condition of equality of the shock velocities $c, \beta$, and $U$ :

$$
F_{3}\left(U_{3}, c^{*}\right)=F_{4}\left(U_{4}, c_{2}\right)=0 .
$$

Point 4 is connected to point $R$ by $U$-shock:

$$
\frac{F_{4}\left(U_{4}, c_{2}\right)-F_{i}\left(U_{R}, c_{2}\right)}{U_{4}-U_{i}}=\frac{-s_{4} f^{R}+s^{R} f_{4}}{f^{R}-f_{4}}=-s^{R} .
$$




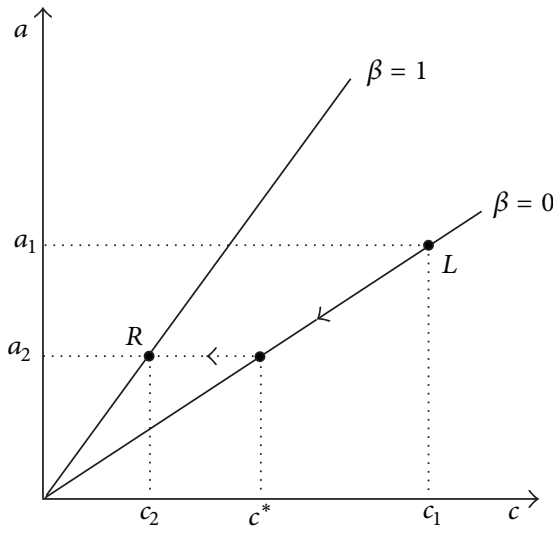

(a)

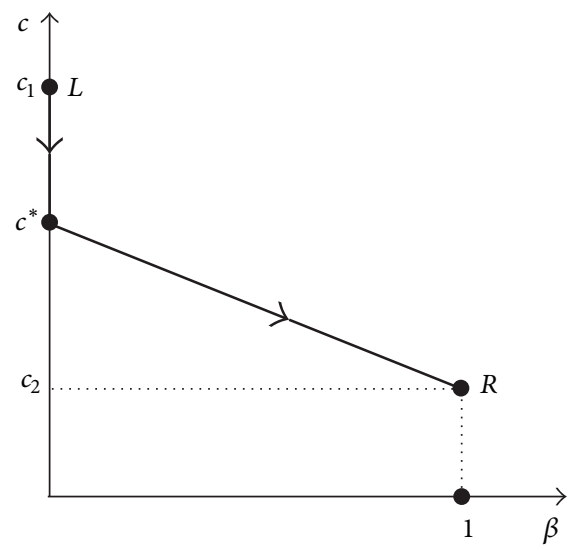

(b)

FIGURE 6: Solution of the auxiliary problem. (a) Adsorption isotherm for chemical for different water salinities and the Riemann problem solution; (b) Riemann problem solution on the plane of chemical concentration $c$ and salinity $\beta$.

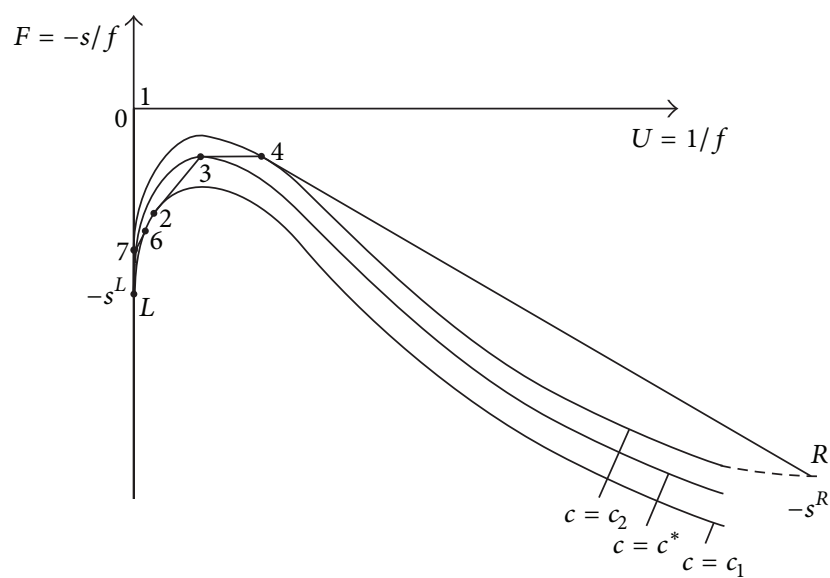

FIgURE 7: The image of the solution in $(F-U)$ plane.

The solution of the Riemann problem equations (28)-(30) with free variables $(x, \varphi)$ is given by the following formulae:

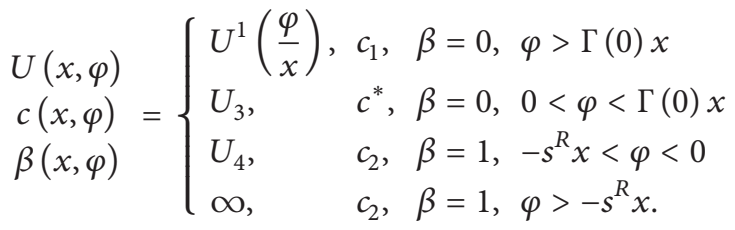

The expression $s=-U F(U, c)$ allows calculating saturation $s(x, \varphi)$ :

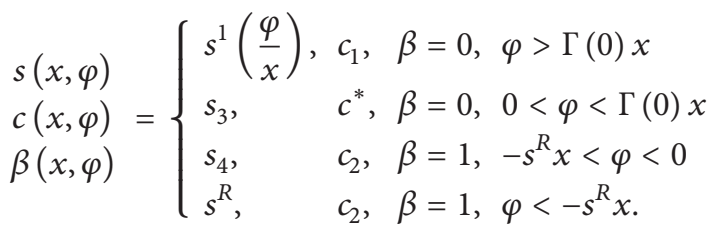

Figure 8 shows the solution of the system $(28)-(30)$ in $(\varphi, x)$ plane. For $\varphi<1$, the solution is self-similar; the wave interaction occurs at $\varphi>1$.

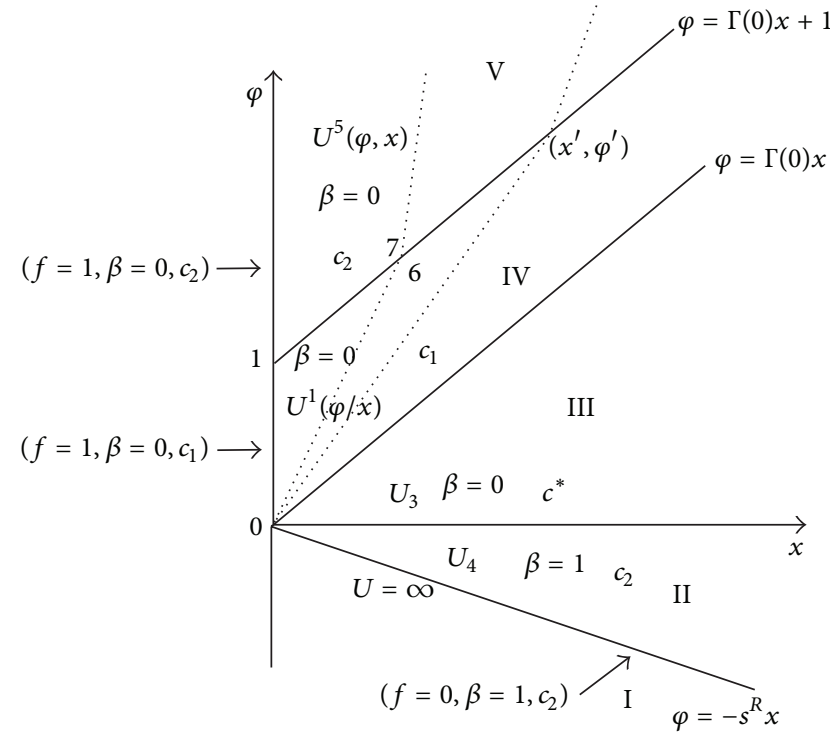

Figure 8: Solution of the auxiliary and lifting system for slug problem in $(\varphi, x)$-plane.

4.3. Inverse Mapping: Change of Variables from $(\varphi, x)$ to $(t, x)$. Time $t=t(x, \varphi)$ for solution is calculated from (12) along any path from point $(x, \varphi)$ to point $(0,0)$. The expression for time $t$ in zone II is

$$
t=\frac{1}{f_{4}} \int_{0}^{\varphi} d \varphi+\frac{s_{4}}{f_{4}} \int_{0}^{x} d x=\left(\frac{-s^{R}+s_{4}}{f_{4}}\right) x .
$$

The expression for time $t$ in zone III is

$$
t=\frac{1}{f_{3}} \int_{0}^{\varphi} d \varphi+\frac{s_{3}}{f_{3}} \int_{0}^{x} d x=\frac{s_{3}}{f_{3}} x .
$$




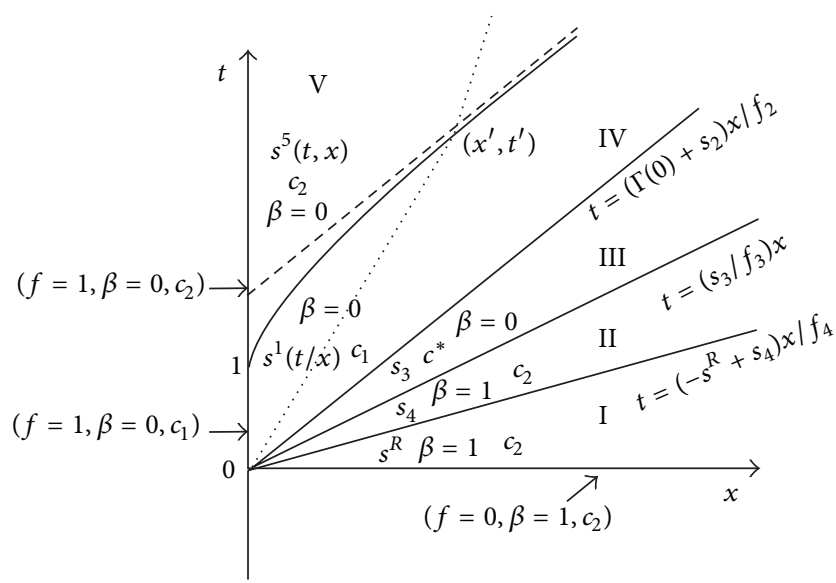

FIGURE 9: Non-self-similar solution of the problem for wave interactions in $(x, t)$-plane.

In zone IV, integral for calculation time, $t=\int_{0,0}^{x, \varphi}(d \varphi / f+$ $s d x / f$ ) is calculated along the characteristic in centred $U$ wave:

$$
t=\frac{\varphi}{f\left(s^{1}(\varphi / x), c_{1}\right)}+\frac{s^{1}(\varphi / x)}{f\left(s^{1}(\varphi / x), c_{1}\right)} x
$$

Figure 9 shows the solution for the Riemann problem at $t<1$; see Figure 10 for detailed description of the Riemann solution and profiles of unknown functions. Finally, the solution of the Riemann problem for the system (4)-(6) is

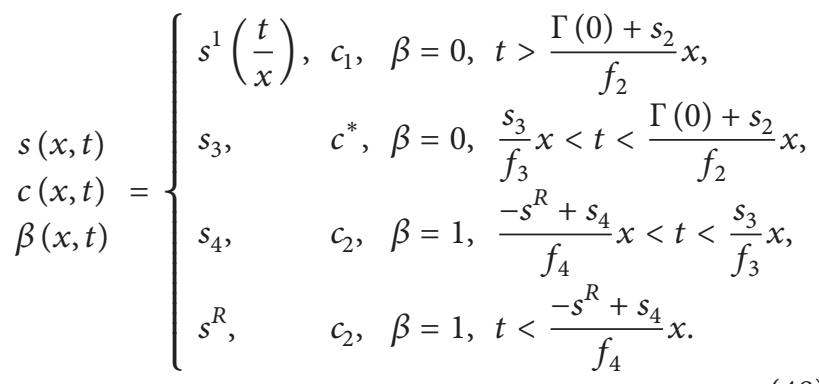

\section{Solution of the Slug Problem}

Now let us solve the slug problem equations (31) and (32) for auxiliary system (29) and (30). The solution of Riemann problem at the point $(0,1)$ is given by $c$-shock with $c^{-}=c_{2}$ and $c^{+}=c_{1}$ under constant $\beta$ :

$$
c(x, \varphi)=\left\{\begin{array}{lll}
c_{2}, & \beta=0, & \varphi>\Gamma(0) x+1 \\
c_{1}, & \beta=0, & \Gamma(0) x<\varphi<\Gamma(0) x+1 \\
c^{*}, & \beta=0, & 0<\varphi<\Gamma(0) x \\
c_{2}, & \beta=1, & -s^{R} x<\varphi<0 .
\end{array}\right.
$$

The solution of the auxiliary system is given by (49).

So, zone I in Figure 8 corresponds to initial conditions, the solution is given by point 4 in zone II, and point 3 holds (a)

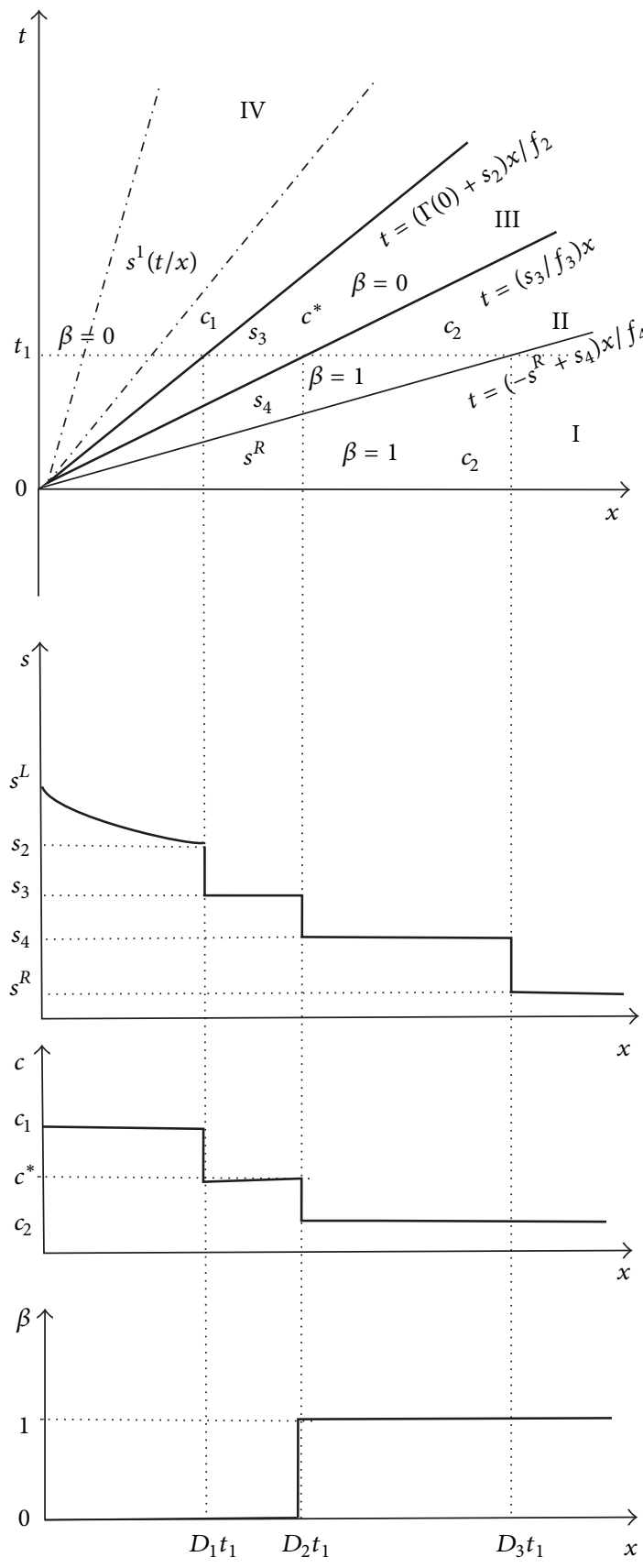

FIgURE 10: Solution of the Riemann problem: (a) trajectories of shock fronts and characteristic lines in $(x, t)$-plane; (b) saturation profile; (c) chemical concentration profile; (d) salinity profile.

in zone III. Centred waves equation (39) fills in zone IV. In zone $\mathrm{V}, c=c_{2}$ and $\beta=0$.

Now let us solve the lifting equation (28) with given $c(x, \varphi)$ and $\beta(x, \varphi)$.

The Hugoniot-Rankine condition for the rear slug front is

$$
\frac{F\left(U^{+}, c_{1}\right)-F\left(U^{-}, c_{2}\right)}{U^{+}-U^{-}}=\Gamma(0) .
$$


$U$ is constant along the characteristic lines behind the rear front

$$
U_{5}(x, \varphi)=U^{-}\left(x^{\prime}, \varphi^{\prime}\right)
$$

where point $\left(x^{\prime}, \varphi^{\prime}\right)$ is located on the rear front and is located on the same characteristic line with point $(x, \varphi)$ :

$$
\frac{\varphi-\varphi^{\prime}}{x-x^{\prime}}=\frac{\partial F\left(U_{5}, c_{1}\right)}{\partial U} .
$$

The solution of lifting equation $U(x, \varphi)$ is given by different formulae in zones I-V:

$$
\begin{aligned}
& U(x, \varphi) \\
& c(x, \varphi) \\
& \beta(x, \varphi) \\
& \quad=\left\{\begin{array}{lll}
U_{5}(x, \varphi), & c_{2}, \quad \beta=0, \varphi>\Gamma(0) x+1 \\
U^{1}\left(\frac{\varphi}{x}\right), & c_{1}, \quad \beta=0, \quad \Gamma(0) x<\varphi<\Gamma(0) x+1 \\
U_{3}, & c^{*}, \quad \beta=0, \quad 0<\varphi<\Gamma(0) x \\
U_{4}, & c_{2}, \quad \beta=1,-s^{R} x<\varphi<0 \\
\infty, & c_{2}, \quad \beta=1, \varphi>-s^{R} x,
\end{array}\right.
\end{aligned}
$$

where the equation for rear front of the chemical slug in the auxiliary plane is

$$
\varphi=\Gamma(0) x+1
$$

Finally, the solution of auxiliary problem equation (53) allows calculating $t(x, \varphi)$ for zones I, II, .., V. Let us start with determining time $t$ along the rear front of the slug. The centred $s$-wave propagates ahead of the rear front

$$
\frac{\varphi}{x}=\frac{f\left(s^{+}, c_{1}\right)-s^{+} f^{\prime}\left(s^{+}, c_{1}\right)}{f^{\prime}\left(s^{+}, c_{1}\right)} .
$$

From (54), (55) follow the expression for $x_{D}\left(\varphi_{D}\right)$ in a parametric form:

$$
\begin{gathered}
x_{D}\left(s^{+}\right)=\frac{f^{\prime}\left(s^{+}, c_{1}\right)}{f\left(s^{+}, c_{1}\right)-f^{\prime}\left(s^{+}, c_{1}\right)\left(s^{+}+\Gamma\right)}=\frac{f^{\prime}\left(s^{+}, c_{1}\right)}{\Delta} \\
\varphi_{D}\left(s^{+}\right)=\frac{f\left(s^{+}, c_{1}\right)-s^{1^{+}} f^{\prime}\left(s^{+}, c_{1}\right)}{f\left(s^{+}, c_{1}\right)-f^{\prime}\left(s^{+}, c_{1}\right)\left(s^{+}+\Gamma\right)} \\
=\frac{f\left(s^{+}, c_{1}\right)-s^{1^{+}} f^{\prime}\left(s^{+}, c_{1}\right)}{\Delta} .
\end{gathered}
$$

Integration of the form (41) along the rear front gives

$$
\begin{aligned}
& t_{D}=\frac{\varphi}{f\left(s^{+}(\varphi, x), c_{2}\right)}+\frac{s^{+}(\varphi, x)}{f\left(s^{+}(\varphi, x), c_{2}\right)} x \\
& t_{D}=\frac{1}{f\left(s^{+}, c_{1}\right)-f^{\prime}\left(s^{+}, c_{1}\right)\left(s^{1^{+}}+\Gamma\right)}=\frac{1}{\Delta} .
\end{aligned}
$$

Finally, the solution of the slug problem for the system (4)(6) subject to initial and boundary conditions equations (7) and (8) is (Figure 9)

$$
\begin{aligned}
& s(x, t) \\
& c(x, t) \\
& \beta(x, t)
\end{aligned}
$$

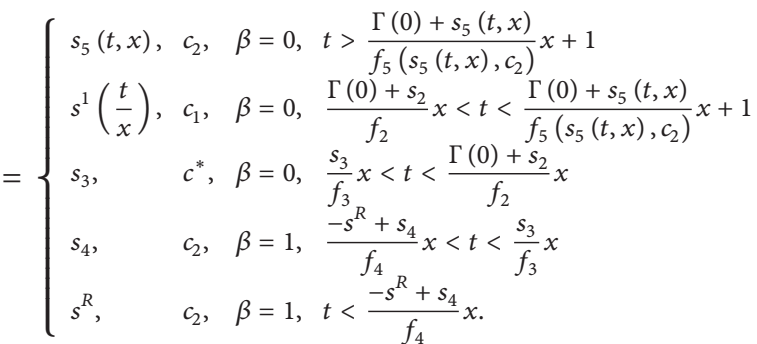

Figure 11 presents trajectories of shock fronts in $(x, t)$-plane along with profiles of unknowns $s, c$, and $\beta$ at typical moments.

Here the trajectory of the rear slug front $x_{D}=x_{D}(t)$ is given in a parametric form (Figure 12)

$$
\begin{gathered}
\frac{1}{x_{D}}=s_{B}+\Gamma(0) \\
\frac{1}{t_{D}}=f_{A},
\end{gathered}
$$

where $s_{B}$ is the abscissa of point $B$ and $f_{A}$ is the ordinate of point $A$ (Figure 12). Equations (60) can be solved graphically. Straight line $A B$ is a tangent to the fractional flow curve $c=c_{1}$, the tangent point in $s+$. The rear front position $x_{D}$ is determined by the interval $\mathrm{BC}$ at the moment determined by AC.

\section{Particular Case for the Polymer Absence in the Reservoir before the Injection}

In reality, there is no chemical initially in the reservoir during the majority of chemical enhanced oil recovery applications; that is, $c(x, 0)=0$. For zero initial polymer concentration, the intermediate polymer concentration is equal to zero, so the points ahead and behind the $\beta$-shock coincide in planes $(c, a)$ and $(s, f)$. The particular simplified solution is (Figures 13 and 14)

$s(x, t)$
$c(x, t)$
$\beta(x, t)$

$$
=\left\{\begin{array}{llll}
s_{5}(t, x), & c=0, & \beta=0, & t>\frac{\Gamma(0)+s_{5}(t, x)}{f_{5}\left(s_{5}(t, x), c_{2}\right)} x+1 \\
s^{1}\left(\frac{t}{x}\right), & c=c_{1}, & \beta=0, & \frac{\Gamma(0)+s_{2}}{f_{2}} x<t<\frac{\Gamma(0)+s_{5}(t, x)}{f_{5}\left(s_{5}(t, x), c_{2}\right)} x+1 \\
s_{3}, & c=0, & \beta=0, & \frac{s_{3}}{f_{3}} x<t<\frac{\Gamma(0)+s_{2}}{f_{2}} x \\
s_{3}, & c=0, & \beta=1, & \frac{-s^{R}+s_{3}}{f_{3}} x<t<\frac{s_{3}}{f_{3}} x \\
s^{R}, & c=0, & \beta=1, & t<\frac{-s^{R}+s_{3}}{f_{3}} x .
\end{array}\right.
$$


(a)

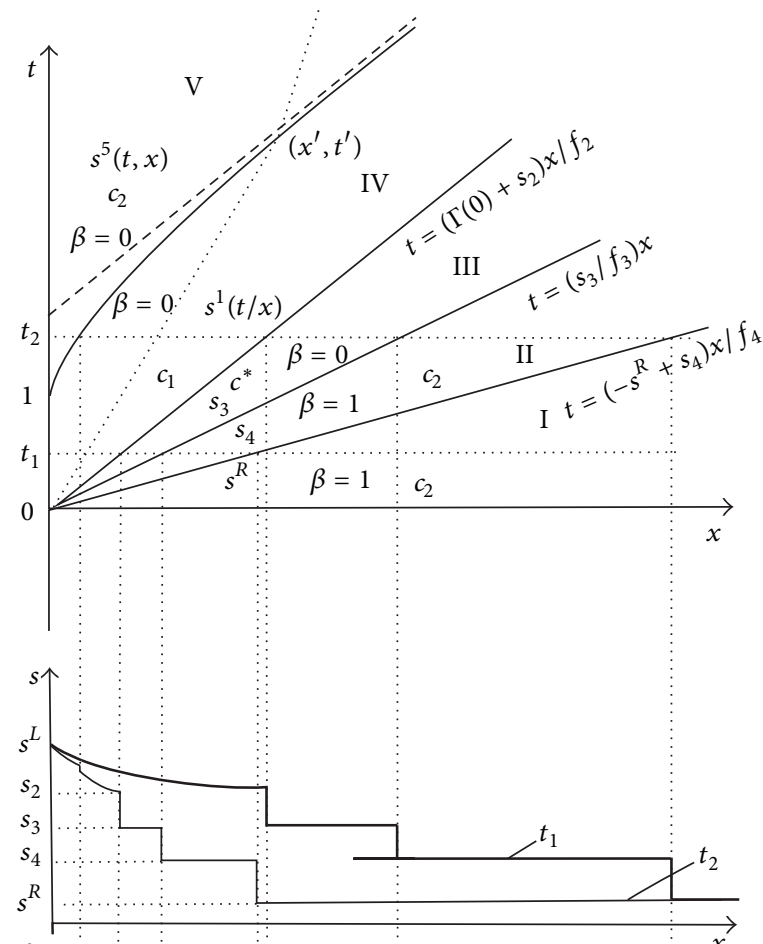

(c)

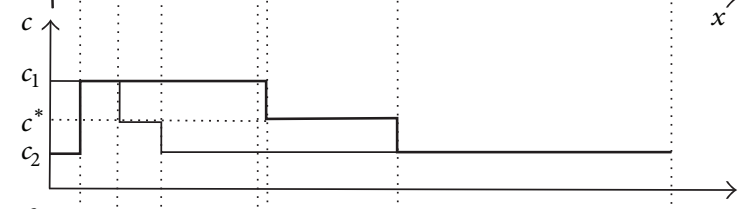

(d)

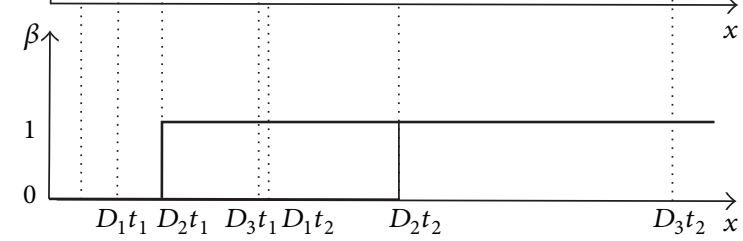

FIGURE 11: The solution of the slug injection problem: (a) trajectories of shock fronts and characteristic lines in $(x, t)$-plane; (b) saturation profile; (c) chemical concentration profile; (d) salinity profile.

\section{Fluid Mechanics Interpretation of the Solution}

Following exact solution equations (4)-(9), let us describe structure of two-phase flow with chemical and salt additives during chemical slug injection.

During continuous injection $t<1$, the solution of chemical slug injection coincides with that of continuous chemical injection. Initial conditions equation (10) is shown by point $R$ that corresponds to low initial saturation and initial concentrations of chemical $c_{2}$ and of salt $\beta=1$. The boundary condition at $x=0$ corresponds to point $L$ of injection of chemical solution with concentration $c_{1}$ and salinity $\beta=0$. The path of Riemann problem solution in plane $(s, f)$ consists of centred $s$-wave with injected chemical concentration and unity salinity, $c$-s-shock $2->3, c, s, \beta$ shock $3->4$, and $s$-shock $4->R$ into initial point (Figure 1). Following nomenclature by Courant and Friedrichs [1] and Lake [9], the Riemann solution is $L-2->3->4->R$. Shock

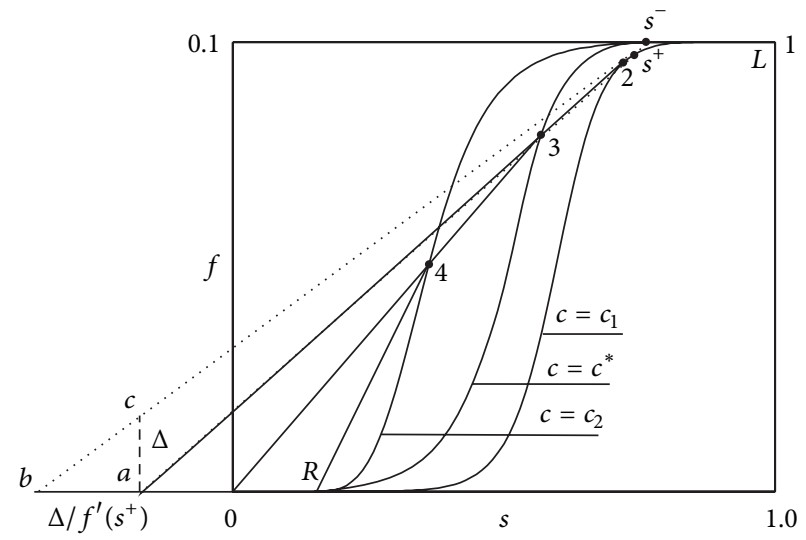

FIGURE 12: Solution of the lifting equation in $(s, f)$-plane.

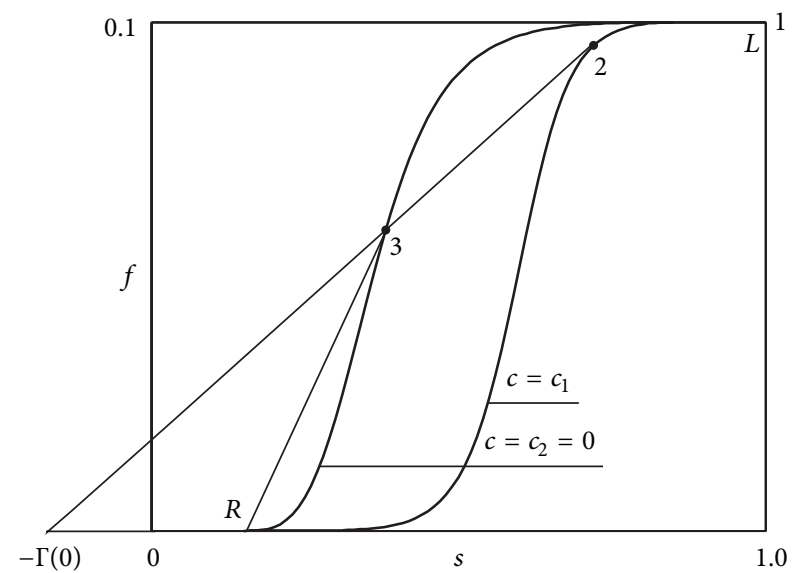

FIGURE 13: Solution of the lifting system in $(f-s)$ plane when $c_{2}=0$.

$2->3$ in plane $(c, \beta)$ is horizontal; shock $3->4$ is vertical (Figure 6(b)). Shock $2->3$ in plane $(c, a)$ occurs along the sorption isotherm; shock $3->4$ is a horizontal jump from isotherm $c=c^{*}$ to that $c=c_{2}$ (Figure 7).

The trajectories of shocks $2->3,3->4$, and $4->R$ are shown in Figure 7. Shock velocities are constant, so the trajectories are straight lines. Let us fix the position $x=1$ of the raw of production wells. Before arrival of the front 4$>R$ at the moment $t=1 / D_{3}$, oil with fraction of water $f^{R}$ and initial concentrations of chemical and salt is produced. After arrival of the front, water-oil mixture with water fraction $f_{4}$ and initial concentrations of chemical and salt is produced until the arrival of the 3->4 front at the moment $t=1 / D_{2}$.

The corresponding profiles of saturation and concentrations are shown in Figure 10. The moment $t_{1}$ for profiles is fixed in Figure 10(a), allowing defining positions of all fronts in this moment. Corresponding profiles at that moment for saturation, chemical concentration, and salinity are shown in Figures 10(b), 10(c), and 10(d), respectively. The saturation profile consists of declining interval $s^{L}-s_{2}$ in $s$-wave, two oilwater banks $s_{3}$ and $s_{4}$, and the initial undisturbed zone $s^{R}$. The chemical concentration profile consists of injected value $c=c_{1}$ in $s$-wave, intermediate value $c^{*}$ in $s_{3}$-bank, and initial 


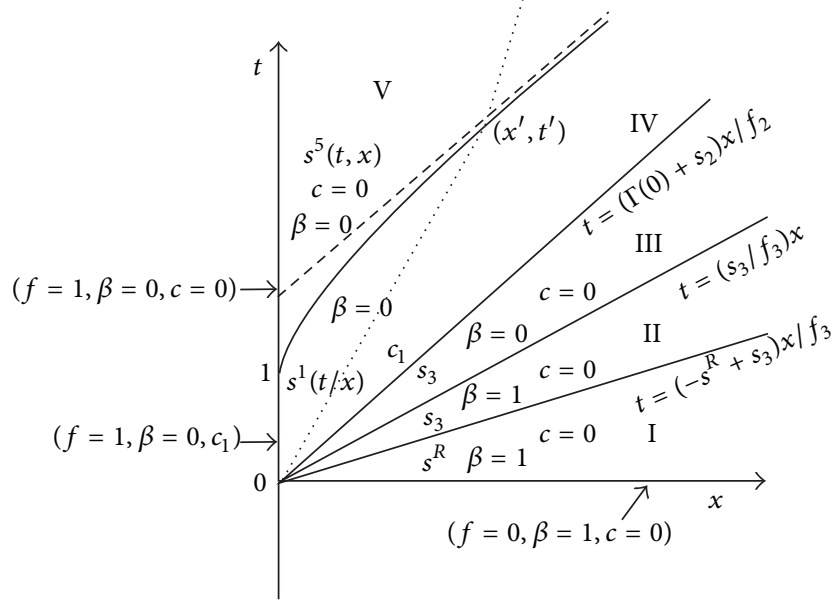

Figure 14: Non-self-similar solution of the problem for wave interactions in $(x, t)$-plane when $c_{2}=0$.

concentration $c_{2}$ in $s_{4}$-bank and in the initial zone. Salinity $\beta$ profile consists of injected value in zones IV and III and initial value in other zones.

Injection of water without chemical and with salinity $\beta=0$ starts at the moment $t=1$. The flow is not selfsimilar anymore. The front trajectories and profiles are shown in Figure 11. The solution for slug problem coincides with the solution for continuous injection ahead of the rear front $x_{D}(t)$. The profiles taken at the moment $t_{1}<1$ during continuous injection (Figure 11) coincide with those from Figure 10.

The propagation of the rear slug front from the beginning of water drive injection in the reservoir is shown in Figure 11(a). The rear front velocity decreases reaching the value of the forward front $D_{3}$ when time tends to infinity. The slug thickness increases and stabilises.

The profiles are shown in Figures 11(b), 11(c), and 11(d) for the moment after the beginning of slug injection $t_{2}>1$. Saturation decreases in a simple wave behind the rear slug front, jumps down on the front, decreases in centred $s$-wave in the slug, and is constant in zones II, II, and I. Chemical slug dissolution during the water drive injection is shown in Figure 11(c). There does occur the full concentration shock from zero behind the read slug front to the injected value in the slug. Further in the reservoir, there does appear a zone of intermediate chemical concentration $c^{*}$ in the bank $s_{3}$. The concentration is equal to its initial value in banks $s_{4}$ and in the initial zone. So, dissolution of slug occurs in the initial water by formation of oil-water bank $s_{3}$ with lower chemical concentration. Salinity changes by full shock on the front between zones III and II.

\section{Conclusions}

Application of the splitting method to $3 \times 3$ conservation law system describing two-phase four-component flow in porous media allows drawing the following conclusions.
(1) The method of splitting between hydrodynamics and thermodynamics in system of two-phase multicomponent flow in porous media allows obtaining an exact solution for non-self-similar problem of displacement of oil by chemical slug with different water salinity for the case of linear polymer adsorption affected by water salinity.

(2) The solution consists of explicit formulae for water saturation and polymer and salt concentrations in the continuity domains and of implicit formulae for front trajectories.

(3) First integrals for front trajectories allow for graphical interpretation at the hodograph plane, yielding a graphical method for finding the front trajectories.

(4) For linear sorption isotherms, the solution depends on three fractional flow curves that correspond to initial reservoir state $R$, injected fluid $L$, and an intermediate curve for intermediate polymer concentration and injected salinity; the value for intermediate polymer concentration is the part of exact solution.

(5) For linear sorption isotherms, the only continuous wave is $s$-wave with constants $c$ and $\beta$; concentrations $c$ and $\beta$ change only across the fronts by jumps; thus the solution of any problem with piece-wise constant initial and boundary conditions is reduced to interactions between $s$-waves and shocks.

(6) Introduction of salinity dependency for sorption of the chemical introduces the intermediate $(c, \beta)$-shock into the solution of the Riemann problem; this shock interacts with $s$-wave and concentration shocks in the solution of any problem with piece-wise constant initial and boundary conditions.

(7) The exact solution shows that the injected chemical slug dissolves in the connate reservoir water rather than in the chemical-free water injected after the slug.

\section{Conflict of Interests}

The authors declare that there is no conflict of interests regarding the publication of this paper.

\section{Acknowledgments}

Long-term cooperation in hyperbolic systems and fruitful discussions with Professors A. Shapiro (Technical University of Denmark), Y. Yortsos (University of Southern California), A. Roberts (University of Adelaide), A. Polyanin (Russian Academy of Sciences), M. Lurie, and V. Maron (Moscow Oil and Gas Gubkin University) are gratefully acknowledged. The reviewers are gratefully acknowledged for their critical comments yielding to significant improvement of the text.

\section{References}

[1] R. Courant and K. O. Friedrichs, Supersonic Flow and Shock Waves, Springer, New York, NY, USA, 1976. 
[2] I. M. Gel'fand, "Some problems in the theory of quasi-linear equations," Uspekhi Matematicheskikh Nauk, vol. 14, no. 2, pp. 87-158, 1959.

[3] A. G. Kulikovskii, N. V. Pogorelov, and A. Yu. Semenov, Mathematical Aspects of Numerical Solution of Hyperbolic Systems, vol. 118, Chapman \& Hall/CRC Press, Boca Raton, Fla, USA, 2001.

[4] B. L. Rozhdestvenski and N. N. Ianenko, Systems of Quasilinear Equations and Their Application to the Dynamics of Gases, vol. 55, American Mathematical Society, 1983.

[5] G. B. Whitham, Linear and Nonlinear Waves, vol. 42, John Wiley \& Sons, Hoboken, NJ, USA, 2011.

[6] A. Kulikovskii and E. Sveshnikova, Nonlinear Waves in Elastic Media, CRC Press, Boca Raton, Fla, USA, 1995.

[7] J. D. Logan, An Introduction to Nonlinear Partial Differential Equations, vol. 93, John Wiley \& Sons, Hobokon, NJ, USA, 2010.

[8] H. K. Rhee, R. Aris, and N. R. Amundson, First-Order Partial Differential Equations: Theory and Application of Hyperbolic Systems of Quasilinear Equations, Dover, New York, NY, USA, 2001.

[9] L. W. Lake, Enhanced Oil Recovery, Prentice Hall, Englewood Cliffs, NJ, USA, 1989.

[10] P. Bedrikovetsky, Mathematical Theory of Oil and Gas Recovery: With Applications to Ex-USSR Oil and Gas Fields, Kluwer Academic Publishers, Boston, Mass, USA, 1993.

[11] T. Johansen, A. Tveito, and R. Winther, "A Riemann solver for a two-phase multicomponent process," SIAM Journal on Scientific and Statistical Computing, vol. 10, no. 5, pp. 846-879, 1989.

[12] T. Johansen and R. Winther, "The Riemann problem for multicomponent polymer flooding," SIAM Journal on Mathematical Analysis, vol. 20, no. 4, pp. 908-929, 1989.

[13] R. Johns and F. M. Orr Jr., "Miscible gas displacement of multicomponent oils," SPE Journal, vol. 1, pp. 39-50, 1996.

[14] F. M. Orr Jr., Theory of Gas Injection Processes, Tie-Line Publications, Copenhagen, Denmark, 2007.

[15] P. Bedrikovetsky, "Displacement of oil by a chemical slug with water drive," Journal of Fluid Dynamics, vol. 3, pp. 102-111, 1982.

[16] V. G. Danilov and D. Mitrovic, "Smooth approximations of global in time solutions to scalar conservation laws," Abstract and Applied Analysis, vol. 2009, Article ID 350762, 26 pages, 2009.

[17] F. Fayers, "Some theoretical results concerning the displacement of a viscous oil by a hot fluid in a porous medium," Journal of Fluid Mechanics, vol. 13, pp. 65-76, 1962.

[18] E. L. Claridge and P. L. Bondor, "A graphical method for calculating linear displacement with mass transfer and continuously changing mobilities," SPE Journal, vol. 14, no. 6, pp. 609-618, 1974.

[19] G. J. Hirasaki, "Application of the theory of multicomponent, multiphase displacement to three-component, two-phase surfactant flooding," SPE Journal, vol. 21, no. 2, pp. 191-204, 1981.

[20] G. A. Pope, L. W. Lake, and F. G. Helfferich, "Cation exchange in chemical flooding-part 1: basic theory without dispersion," SPE Journal, vol. 18, no. 6, pp. 418-434, 1978.

[21] G. I. Barenblatt, V. M. Entov, and V. M. Ryzhik, Theory of Fluid Flows through Natural Rocks, Kluwer Academic Publishers, London, UK, 1989.

[22] G. S. Braginskaya and V. M. Entov, "Nonisothermal displacement of oil by a solution of an active additive," Fluid Dynamics, vol. 15, no. 6, pp. 873-880, 1980.
[23] O. Dahl, T. Johansen, A. Tveito, and R. Winther, "Mulicomponent chromatography in a two phase environment," SIAM Journal on Applied Mathematics, vol. 52, no. 1, pp. 65-104, 1992.

[24] T. Johansen, Y. Wang, F. M. Orr Jr., and B. Dindoruk, "Fourcomponent gas/oil displacements in one dimension-part I: global triangular structure," Transport in Porous Media, vol. 61, no. 1, pp. 59-76, 2005.

[25] T. Johansen and R. Winther, "The solution of the Riemann problem for a hyperbolic system of conservation laws modeling polymer flooding," SIAM Journal on Mathematical Analysis, vol. 19, no. 3, pp. 541-566, 1988.

[26] P. Bedrikovetsky and M. Chumak, "Riemann problem for two-phase four-and morecomponent displacement (Ideal Mixtures)," in Proceedings of the 3rd European Conference on the Mathematics of Oil Recovery, 1992.

[27] V. Entov, F. Turetskaya, and D. Voskov, "On approximation of phase equilibria of multicomponent hydrocarbon mixtures and prediction of oil displacement by gas injection," in Proceedings of the 8th European Conference on the Mathematics of Oil Recovery, 2002.

[28] V. Entov and D. Voskov, "On oil displacement by gas injection," in Proceedings of the 7th European Conference on the Mathematics of Oil Recovery, 2000.

[29] R. T. Johns, B. Dindoruk, and F. M. Orr Jr., "Analytical theory of combined condensing/vaporizing gas drives," SPE Advanced Technology Series, vol. 1, no. 2, pp. 7-16, 1993.

[30] C. Wachmann, "A mathematical theory for the displacement of oil and water by alcohol," Old SPE Journal, vol. 4, pp. 250-266, 1964.

[31] A. Zick, "A combined condensing/vaporizing mechanism in the displacement of oil by enriched gases," in Proceedings of the SPE Annual Technical Conference and Exhibition, 1986.

[32] O. M. Alishaeva, V. M. Entov, and A. F. Zazovskii, "Structures of the conjugate saturation and concentration discontinuities in the displacement of oil by a solution of an active material," Journal of Applied Mechanics and Technical Physics, vol. 23, no. 5, pp. 675-682, 1982.

[33] S. Geiger, K. S. Schmid, and Y. Zaretskiy, "Mathematical analysis and numerical simulation of multi-phase multi-component flow in heterogeneous porous media," Current Opinion in Colloid and Interface Science, vol. 17, no. 3, pp. 147-155, 2012.

[34] K. S. Schmid, S. Geiger, and K. S. Sorbie, "Analytical solutions for co- and counter-current imbibition of sorbing, dispersive solutes in immiscible two-phase flow," in Proceedings of the 12th European Conference on the Mathematics of Oil Recovery, 2012.

[35] A. P. Pires, P. G. Bedrikovetsky, and A. A. Shapiro, "A splitting technique for analytical modelling of two-phase multicomponent flow in porous media," Journal of Petroleum Science and Engineering, vol. 51, no. 1-2, pp. 54-67, 2006.

[36] A. P. Pires, Splitting between thermodynamics and hydrodynamics in the processes of enhanced oil recovery [Ph.D. thesis], Laboratory of Petroleum Exploration and Production, North Fluminense State University UENF, 2003.

[37] H.-K. Rhee, R. Aris, and N. R. Amundson, "On the theory of multicomponent chromatography," Philosophical Transactions of the Royal Society A, vol. 267, no. 1182, pp. 419-455, 1970.

[38] V. M. Entov and A. F. Zazovskii, "Displacement of oil by a solution of an active and a passive additive," Fluid Dynamics, vol. 17, no. 6, pp. 876-884, 1982.

[39] C. B. Cardoso, R. C. A. Silva, and A. P. Pires, "The role of adsorption isotherms on chemical-flooding oil recovery," 
in Proceedings of the SPE Annual Technical Conference and Exhibition (ATCE '07), pp. 773-780, November 2007.

[40] B. Vicente, V. Priimenko, and A. Pires, "Streamlines simulation of polymer slugs injection in petroleum reservoirs," in Proceedings of the SPE Latin America and Caribbean Petroleum Engineering Conference, 2012.

[41] S. Oladyshkin and M. Panfilov, "Splitting the thermodynamlics and hydrodynamics in compositional gas-liquid flow through porous reservoirs," in Proceedings of the 10th European Conference on the Mathematics of Oil Recovery, 2006.

[42] P. M. Ribeiro and A. P. Pires, "The displacement of oil by polymer slugs considering adsorption effects," in Proceedings of the SPE Annual Technical Conference and Exhibition (ATCE '08), pp. 851-865, September 2008.

[43] P. Bedrikovetski, A. Pires, and A. Shapiro, "Conservation law system for two-phase n-component flow in porous media: splitting between thermodynamics and hydrodynamics," in Proceedings of the 10th International Congress on Hyperbolic Problems Theory, 2004.

[44] A. Pires, P. Bedrikovetsky, and A. Shapiro, "Splitting between thermodynamics and hydrodynamics in compositional modelling," in Proceedings of the 9th European Conference on the Mathematics of Oil Recovery, 2004. 


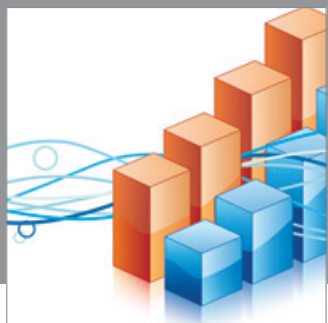

Advances in

Operations Research

mansans

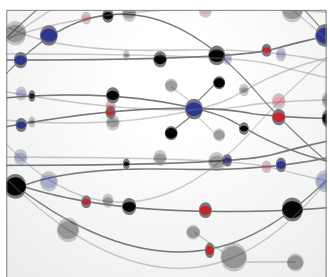

The Scientific World Journal
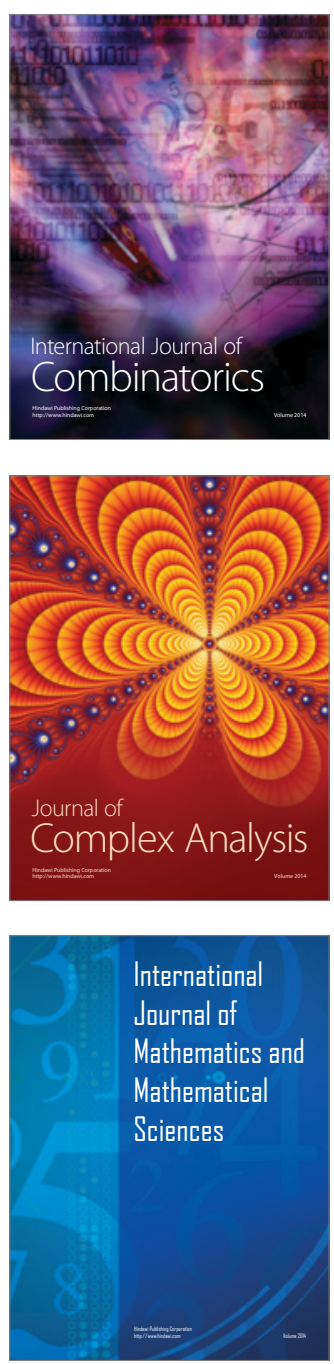
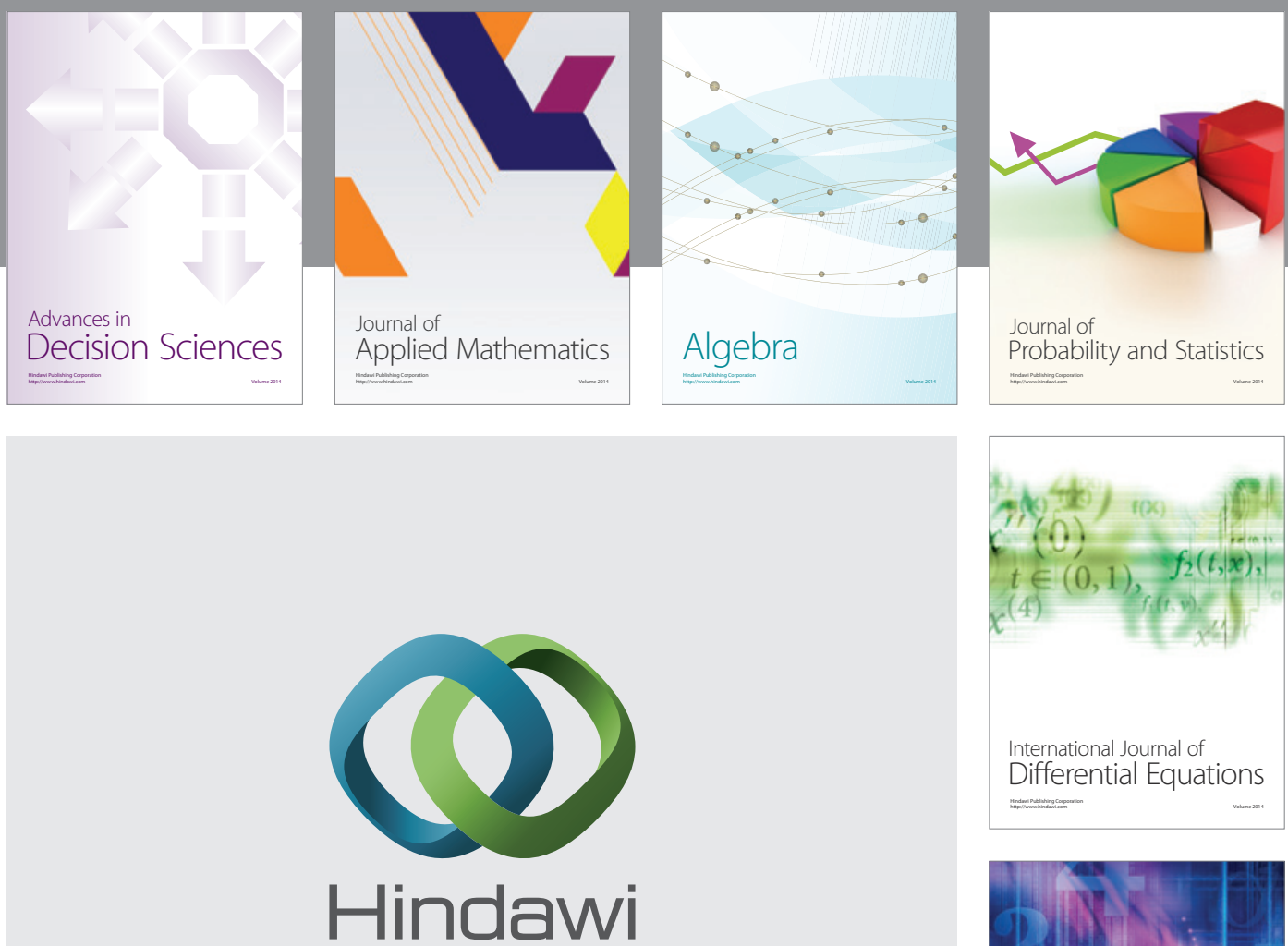

Submit your manuscripts at http://www.hindawi.com
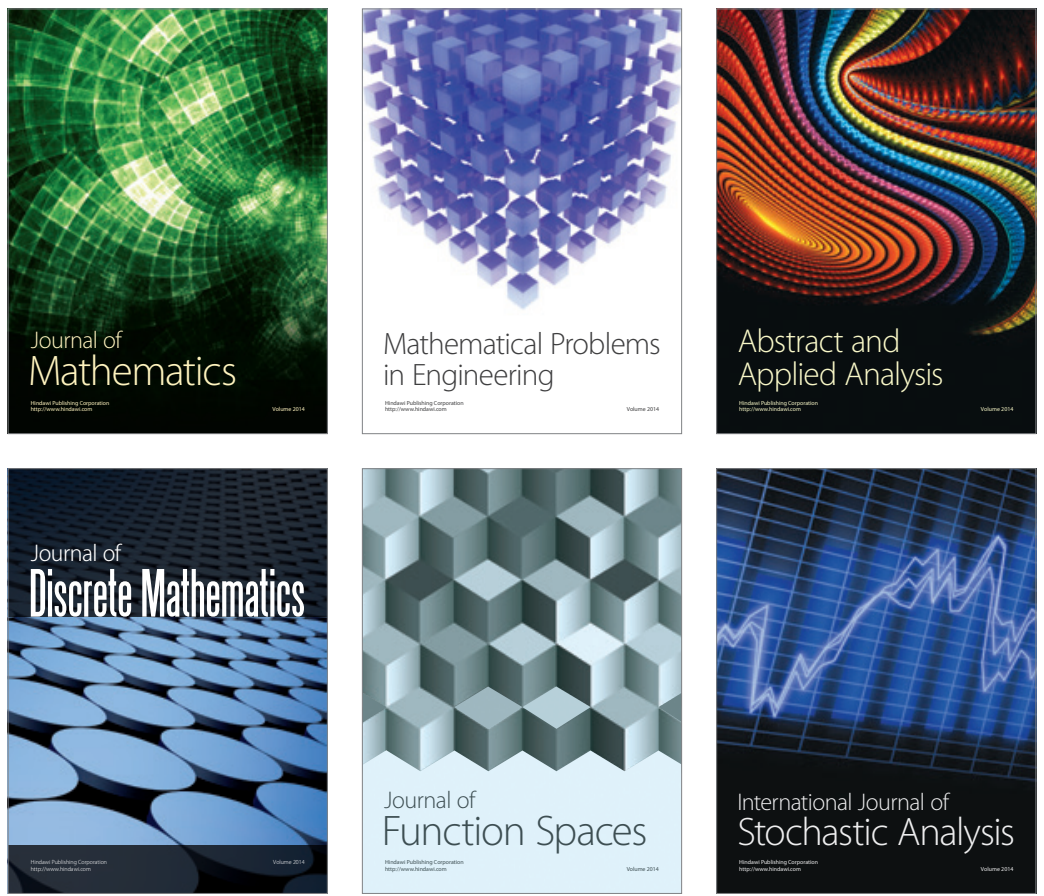

Journal of

Function Spaces

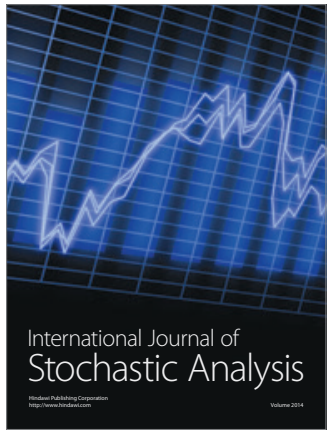

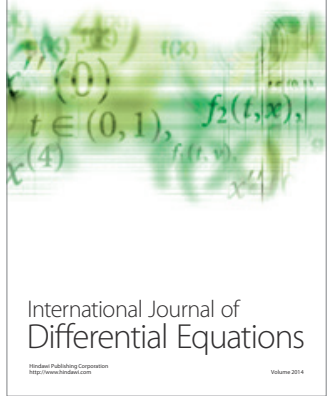
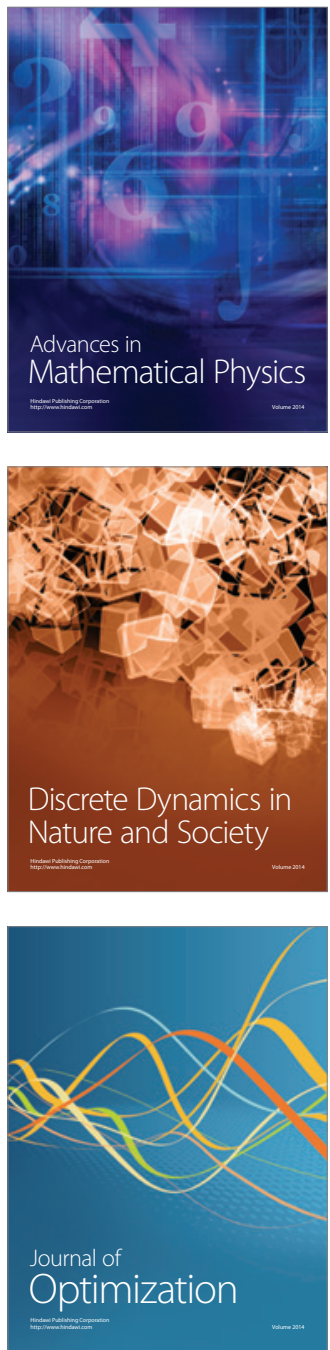